Archives

Du cahier de la coutume ... au livre // Études urbaines

\title{
Quelques réflexions à propos des commissaires du roi dans la rédaction et la réformation des coutumes $\mathrm{au} \mathrm{XVI} \mathrm{e}^{\mathrm{e}}$ siècle
}

\section{Robert Descimon}

\section{(2) OpenEdition}

\section{Journals}

Édition électronique

URL : http://journals.openedition.org/ccrh/1393

DOI : $10.4000 /$ ccrh. 1393

ISSN : 1760-7906

Éditeur

Centre de recherches historiques - EHESS

Édition imprimée

Date de publication : 20 avril 2001

ISSN : 0990-9141

Référence électronique

Robert Descimon, «Quelques réflexions à propos des commissaires du roi dans la rédaction et la réformation des coutumes au XVI e siècle », Les Cahiers du Centre de Recherches Historiques [En ligne], 26 | 2001, mis en ligne le 30 novembre 2008, consulté le 20 avril 2019. URL : http:// journals.openedition.org/ccrh/1393; DOI : 10.4000/ccrh.1393

Ce document a été généré automatiquement le 20 avril 2019.

Article L.111-1 du Code de la propriété intellectuelle. 


\title{
Quelques réflexions à propos des commissaires du roi dans la rédaction et la réformation des coutumes au XVI ${ }^{\mathrm{e}}$ siècle
}

\author{
Robert Descimon
}

1 Lors des opérations de rédaction et de réformation des coutumes ${ }^{1}$, les commissaires $d u$ roi tiennent un rôle irremplaçable. Pour la plupart, il s'agissait de magistrats des parlements. Mais de magistrats choisis par le roi, c'est-à-dire ses conseils, même s'il est permis de douter qu'Henri II, sa veuve, ses fils et leurs ministres aient porté un grand intérêt à cette besogne. La confiance royale était-elle acquise aux commissaires en fonction de traits particuliers qui les auraient distingués des autres juges? S'interroger sur la culture de ces hommes, spécialement leur culture juridique, sur leur pratique, sur leurs relations réciproques et leur insertion dans des réseaux peut permettre d'apporter quelques éléments de réponse à des questions que les juristes n'ont pas laissées de côté, mais qui ne seront pas résolues tant qu'on ne disposera pas d'un cadre sociologique général qui permette de qualifier la magistrature dans son ensemble².

\section{Le partage inégal d'une culture}

2 1. Les commissaires sont présentés par leurs lettres de commission comme des hommes du roi, au moins temporairement investis d'une mission de confiance dont la technicité écartait d'autres agents royaux, de robe courte par exemple, plus volontiers employés par les Valois dans leurs affaires les plus importantes. À l'époque, la noblesse personnelle des grands magistrats des cours souveraines n'en faisait pas des nobles de robe, mais des représentants éminents des élites citadines, même si la théorie voulait encore que les juges souverains aient été tirés de chacun des trois états qu'ils représentaient donc. Naturellement, les témoignages abondent sur la méfiance qu'inspiraient les juristes savants à toutes les autres couches de la population. Leur rôle d'intercesseurs était 
cependant assez volontiers reconnu par l'ensemble des sujets dans la mesure où ils exerçaient une médiation indispensable dans le règlement des conflits exacerbés par les troubles qui avaient marqué la société du Moyen Âge tardif ${ }^{3}$. Un consensus social venait donc ainsi renforcer le choix politique du souverain.

3 L'opération de rédaction royalisait pour ainsi dire les usages locaux. Elle répondait aux attentes des sujets dans la mesure où elle améliorait ce qu'on appelle aujourd'hui la sécurité juridique. L'enquête par turbes que la rédaction rendait inutile ou très exceptionnelle, était une procédure longue et aléatoire. La coutume, dans son oralité, ne formait pas un système juridique stabilisét. L'œuvre de rédaction, souhaitée par les justiciables, avait néanmoins un coût symbolique pour eux dans le contexte du renforcement de l'autorité monarchique après Charles VII : les usages locaux tiraient désormais leur force de la seule autorité souveraine, source de toute loi ${ }^{5}$. Il n'est pas étonnant que l'ordonnance de Montilz-lez-Tours (1454) n'ait pas reçu vraiment d'application avant Louis XII et François I ${ }^{\mathrm{er}}$, rois respectés et forts. Mais le nom de Louis XII, père du peuple, est plus spécialement attaché à l'œuvre de rédaction. C'est que l'opération supposait la consultation des sujets, sujets des trois ordres qui concluaient, sur leurs droits respectifs, un accord dans lequel le roi et ses commissaires n'intervenaient théoriquement que pour le sanctionner.

4 Une première hypothèse ressort de ces considérations préliminaires. Les commissaires agissaient conformément à leur culture politique qui en faisait les hérauts du pouvoir absolu des rois et les protagonistes de son renforcement pratique. Les magistrats français, juristes savants, n'avaient sans doute pas beaucoup plus de sympathie spontanée pour les droits des rustiques que leurs confrères du reste de l'Europe continentale ${ }^{6}$. Mais la royalisation de ces droits informait une double conversion culturelle: si les populations acceptaient que la source et la sanction de leurs coutumes devinssent royales dans leur principe et, en pratique, parlementaires, les magistrats des parlements faisaient leur ce droit, précisément dans la mesure où il devenait royal. Ils le tinrent donc pour un droit savant qui leur appartenait au même titre que le droit romain et qu'il fallait commenter en latin pour manifester la distance qui s'était créée par la rédaction entre les praticiens des sièges et les jurisconsultes, seuls désormais habilités à tenir un discours autorisé sur les coutumes ${ }^{7}$. La rédaction provoquait donc une révolution culturelle dans la land law française. Charles du Moulin fut l'artisan et le symbole de cette mutation: son commentaire du titre des fiefs de la coutume de Paris et les nombreux autres commentaires d'autres coutumes auxquels il travailla marquent une conversion culturelle et politique : le latin commente des textes en langue vulgaire, l'explication des droits féodaux sert de prétexte à une construction stricte des droits du roi investis d'un monopole de légitimité, puisque tous les autres droits et privilèges étaient censés émanés ou délégués de la puissance souveraine qui ne connaissait aucune autre légitimité que la sienne ${ }^{8}$.

5 Une seconde hypothèse apparait en corollaire. L'idéologie générale des commissaires impliquait leur adhésion à un certain modèle d'exercice de la royauté absolue, celui que J. Russell Major a qualifié de représentatif ou de consultatify. Il n'y a là rien d'étonnant à l'époque de Louis XII, quand Claude de Seyssel théorisait une conception approchante du pouvoir monarchique. Il n'en était pas tout à fait de même à partir du règne de François Ier, quand le discours absolutiste fut développé dans des formes intransigeantes par les théoriciens politiques qui, pourtant, ne cessèrent jamais de poser les bornes rationnelles de l'exercice légitime du pouvoir ${ }^{10}$. L'absolutisme à visage humain des 
légistes avait un corollaire : l'hostilité aux droits des seigneurs ${ }^{11}$. Quoique la noblesse n'ait pas été absente des assemblées des trois ordres, elle siégeait plutôt par ses procureurs spéciaux, qui étaient les praticiens ou juristes de leurs justices seigneuriales. Les droits des seigneurs semblent avoir été d'autant plus mal défendus qu'ils n'étaient déjà plus dans l'air du temps au xVI ${ }^{e}$ siècle. Bon nombre paraissaient abusifs aux hommes de loi et les gentilshommes eux-mêmes n'étaient pas immunisés contre ce sentiment général. Bref la rédaction, comme la réformation, fut l'occasion de gigantesques autodafés de droits féodaux et seigneuriaux ${ }^{12}$. Les commissaires y eurent leur grande part. Ils employèrent à leur fin une technique de procédure : ils faisaient passer dans le texte la solution qui avait leur préférence en réservant aux opposants de se pouvoir devant le parlement, espérant qu'ils n'auraient pas l'énergie de poursuivre leurs oppositions ${ }^{13}$. Ainsi la rédaction recouvrit le droit coutumier d'un vernis social unitaire, alors qu'historiquement, il faisait sans doute coexister des normes réservées à la classe seigneuriale des propriétaires fonciers et des règles propres aux roturiers ou coutumiers, car ces deux groupes vivaient dans des systèmes sociaux antagonistes et n'avaient pas besoin des mêmes lois pour assurer leur reproduction sociale ${ }^{14}$.

6 2. Cependant le compromis politique qui sous-tendait les rédactions ne levait pas tout à fait les obstacles culturels que pouvait faire naitre dans les esprits des magistrats l'opération de mise des coutumes par écrit. On a beaucoup glosé, à partir de notations minces, sur les différences de conceptions qui auraient opposé entre eux les commissaires dans leurs travaux pratiques de rédaction. Ainsi la distinction entre les premiers présidents Lizet et de Thou est devenue un passage obligé des exposés sur les coutumes rédigées. Pierre Lizet, parce qu'auvergnat, aurait été si nourri de droit romain qu'il aurait influencé les assemblées des états pour les pousser vers des rédactions à inflexions romanistes. Christofle de Thou, pour sa part, aurait tenu la coutume de Paris comme une matrice générale qui donnait les solutions aux lacunes des coutumes périphériques ${ }^{15}$. Il n'y a aucune raison de mettre en cause ces témoignages, mais leur portée paraît incertaine. Car l'assertion soulève au moins deux interrogations.

7 Contrairement aux jurisconsultes européens, les magistrats français ne peuvent être bien connus du point de vue de leur formation intellectuelle. L'usage d'étudier et de prendre ses grades en Italie, comme le chancelier de L'Hospital, semble avoir battu son plein entre les rédactions, dont la vague se situe de la fin $\mathrm{du} \mathrm{Xv}^{\mathrm{e}}$ siècle aux années 1520 , et les réformations des années 1555-1580. Même durant cette période, le voyage d'Italie resta l'apanage d'une élite de juristes favorisés par leur propre richesse ou la protection de grands seigneurs désireux de s'assurer les services de juristes compétents (comme la comtesse de Nevers pour le fils de son commensal, Charles Lamoignon). Parmi les commissaires de la réformation, Barthélemy Faye était docteur de Padoue. Un tel investissement culturel n'était pas à la portée de la majorité des juges : si, au parlement de Paris, siégeait un bon nombre de docteurs de Bourges, de Poitiers ou d'Orléans, la majorité était constituée de simples licenciés. Le premier président de Thou lui-même avait fait ses études à Poitiers (il était vraisemblablement docteur). Les études de Jacques Viole ne sont pas connues avec précision. C'est le sort commun de la plupart des magistrats qui ont travaillé à la mise en écriture du droit coutumier ${ }^{16}$. D'où le soupçon que le gros des magistrats français n'étaient pas si savants qu'ils le prétendaient et que leurs connaissances en droit commun étaient des souvenirs d'études qu'ils ne rafraîchissaient pas volontiers (la passion de de Thou pour l'étude de l'Antiquité était assez exceptionnelle pour lui valoir des éloges). Le droit romain était en théorie la raison 
écrite, mais c'était une idole adorée qui ne guidait pas forcément la pratique de bien des juristes attachés à la tradition législative et coutumière du royaume de France ${ }^{17}$.

Mais on peut soutenir un point de vue strictement inverse pour aboutir à la même conclusion. La tradition autochtone française de droit savant, i. e. romain, n'avait jamais été négligeable intellectuellement et le triomphe du mos gallicus renforça le prestige des docteurs français ou naturalisés ${ }^{18}$. Barthélemy Faye, recueillit en 1553 des Commentaria juris civilis de François Connan, un élève d'Alciat, dont l'œuvre devint le manifeste des nouveaux romanistes. Le droit romain se trouva ainsi pris dans une vaste entreprise de nationalisation et entrait dans une sorte de compatibilité intellectuelle avec les principes propres au droit français, fût-il coutumier.

Il est donc difficile de comprendre les effets de leur formation universitaire sur les conceptions juridiques des magistrats rédacteurs ou réformateurs. L'opposition entre des tendances romanistes, des tendances coutumières et des tendances royalistes et nationales, pourrait bien être un simple effet rhétorique, facile à manier pour des juristes d'Ancien Régime appelés à évaluer d'un jugement synthétique l'œuvre de leurs grands devanciers qui avaient procédé aux rédactions et aux réformations en puisant dans un vaste répertoire de références culturelles éclectiques.

3. La culture juridique des magistrats semble en effet sortie d'un moule commun, la vera philosophia, qui transcendait les oppositions éventuelles issues de leur formation ou de leurs inclinations personnelles. La legitima scientia du perfectus doctor faisait en quelque sorte le lien entre la pure herméneutique juridique et les sciences morales qui s'imposèrent avec les Lumières. La plasticité de doctrines qui peuvent justifier aussi bien le vivere civile que l'absolutisme législatif démontre assez leur dimension pratique. La science du droit est une praxis. Elle oscille entre un pôle spéculatif de la methodus, la ratio, l'institutio, qui explique la passion momentanée des juristes pour Ramus et sa logique dichotomique, et un pôle pragmatique qui met en scène l'homme de bien, expert en équité et constitué par le roi en dignité. Cet hommes-là, que symbolisait Christofle de Thou, est apte à guider les sujets en tant qu'ils sont des homines politici. Il brille par sa mémoire et son éloquence autant que par sa science. Il est l'artisan d'une justice harmonique (eunomia) dont la coutume, rendant à chacun des trois ordres ce qui lui est dû, peut être une expression pertinente. Alors que dans la doctrine d'une monarchie absolue de droit divin, qui n'est pas encore parfaitement fixée avant la victoire des Bourbons, exclut de penser la loi en termes de contrat, la coutume se définit par le consentement tacite des intéressés, si elle est orale, sur leur consentement explicite, si elle est rédigée, le tout sous condition de la sanction du souverain ${ }^{19}$. Entre la nature, où Dieu est souverain, et la fiction, dont le maitre est le roi comme substitut de Dieu, il y avait place pour une seconde nature, qui est précisément la coutume en liaison avec l'idée d'immémorialité ou de prescription. On propose donc l'hypothèse que les jurisconsultes rédacteurs et réformateurs vivaient dans une représentation relativement unifiée du monde social ${ }^{20}$.

11 4. Enfin, les magistrats rédacteurs ou réformateurs n'auraient su s'abstraire des idées de la justice en cours dans la société en général ${ }^{21}$. Leur rôle de conseils juridiques dans les litiges privés contribuait plus que les procès à faire adopter par les justiciables leurs conceptions propres de professionnels du droit. Le sens de l'équité et les choix de transmission mis en œuvre par les familles ne manquaient pas d'exercer une influence sur les juges, eux-mêmes grands clients des tribunaux: les légistes se trouvaient désormais à la tête de lignages dont ils souhaitaient la perpétuation en ligne paternelle et 
ils modelaient la norme en grande partie en fonction des exigences particulières de leur reproduction sociale ${ }^{22}$. La rédaction des coutumes s'insérait donc dans des pratiques quasi quotidiennes d'échanges et d'acculturations réciproques.

Certaines coutumes, certains usages paraissaient injustes ou mauvais. L'introduction de la représentation en ligne directe, puis en ligne collatérale, l'introduction d'une légitime de droit semblaient des mesures d'équité, au moins parmi les roturiers. Les jurisconsultes le dirent avec force lors des rédactions et des réformations. Mais leur opinion rencontrait le désir des populations et des praticiens qui n'avaient pas un attachement absolu à leurs usages locaux, mais seulement à ce qui leur semblait positivement spécifique dans ces coutumes. Les actes notariés parisiens des années 1500 anticipaient sur l'introduction de la représentation en ligne directe dans la rédaction de 1510, par des stipulations particulières, soit dans les contrats de mariage, soit dans des actes de rappel exprès, du vivant des enfants, quand les successions n'étaient pas encore ouvertes. Moins systématiquement, il en était de même dans les années 1560-1570 (testaments, contrats de mariages) pour la représentation en ligne collatérale et la légitime avant la réformation de $1580^{23}$. Les conceptions des justiciables et des juges étaient dominées par une communauté d'esprit qui explique d'ailleurs la capacité des populations à faire un usage tactique des règles de droit qu'elles transformaient en des instruments adaptés à leur projet personnel de vie et de transmission, avec l'accord tacite des cours de justice, tant que la lettre du droit n'était pas violée. Des normes dominantes et des conflits de normes secondaires formaient ainsi l'ambiance générale où se déroulaient les opérations de rédaction et de réformation.

Cet accord relatif sur un système de normes est peut-être un épisode relativement bref (les trois premiers quarts du XvI ${ }^{\mathrm{e}}$ siècle) dans l'histoire juridique de la France. Il semble probable que les conflits de normes devinrent plus durs avec le triomphe d'une conception de la loi positive comme pure expression de la volonté du roi absolu. La République de Jean Bodin (1576) marqua en la matière un tournant. Le règne d'Henri III et plus encore les temps des premiers Bourbons, sans parler du règne personnel de Louis XIV, n'offraient plus un contexte favorable à des pratiques de consultation où la représentation des sujets pouvait contribuer à l'établissement des normes qui régissaient leurs propres affaires ${ }^{24}$. Encore l'analyse que l'on vient de produire reste-t-elle toute théorique : la pratique des commissaires ne révèle-t-elle pas l'autoritarisme de gens sûrs de leurs savoirs et de leurs pouvoirs?

\section{Travaux pratiques : retour sur le procès de la réformation de la coutume d'Amiens (20 août 1568)}

14 La théorie voudrait que les commissaires se fussent abstenus d'intervenir sur le fond quand les trois ordres du bailliage étaient d'accord. Mais les magistrats avaient des idées très arrêtées sur le juste et l'injuste, ce qui était après tout leur métier. Ils étaient souvent partisans de solutions moyennes et influencés par les décisions de la coutume de Paris qui était la leur en plusieurs sens. René Filhol analyse l'échec de Thou, Faye et Viole dans leur tentative, en 1556, pour passer en force contre l'avis des trois états du bailliage de Châlons en Champagne. Les commissaires s'étaient mis en tête de supprimer le principe d'égalité stricte entre héritiers, qui obligeait les enfants avantagés à rapporter la valeur de leur donation aux partages de leurs père et mère, et de supprimer aussi la noblesse maternelle que la coutume assortissait de cette égalité absolue entre héritiers. Le mandat 
impératif qu'avaient reçu les députés eut raison de la volonté des magistrats. L'affaire est connue par des sources locales, car rien n'a transpiré de l'affrontement dans le procèsverbal officiel. Elle est intéressante aussi bien sur le fond que dans la forme ${ }^{25}$.

Filhol consacre une longue étude ${ }^{26}$ à un événement plus marquant, le procès que durent soutenir les commissaires, toujours les mêmes, un procès que leur intentaient certains représentants éminents du bailliage d'Amiens, tels le cardinal de Créquy, le cardinal de Bourbon, le cardinal de Guise, le duc d'Aumale et le corps de ville d'Amiens. Filhol considérait cette affaire comme un véritable manuel de réformations des coutumes. La coutume, rédigée en 1507, avait été réformée en deux jours, les 23 et 24 septembre 1567. Le procès, plaidé le 20 août 1568 , concernait l'introduction de la représentation en ligne collatérale chez les nobles, à propos de la succession du vidame d'Amiens, Louis d'Ailly, mort sans enfant et laissant une sœur (son unique héritière, si la représentation en ligne collatérale n'avait pas lieu) et des neveux, fils d'un frère prédécédé, qui avaient un intérêt majeur à voir appliquer la coutume réformée. J'ai rouvert le vieux registre qui contient les plaidoiries prononcées à cette occasion ${ }^{27}$. Elles occupent plus d'une centaine de pages : cette transcription narcissique illustre l'importance d'une cause auto-référentielle, une de celles qui construisaient l'autorité du parlement. On ne peut écarter l'idée, en relevant les noms des plaignants et de leurs avocats, que l'entreprise ait été une manifestation d'hostilité du camp ultra-catholique et lorrain (Françoise d'Ailly était la veuve du gouverneur du duché de Guise) envers la politique royale.

L'argumentaire des contestataires soulevait des questions de fait : le peu de temps passé à Amiens par les commissaires, les menaces de guerre (c'était le temps de la surprise de Meaux tentée par Condé contre la Cour) qui diminuèrent la participation des députés à l'assemblée, le non dépôt d'un exemplaire de la coutume réformée au greffe du bailliage d'Amiens (ce qui aurait manifesté qu'une grande partie du travail avait été effectuée à Paris hors de la présence des états); et des arguments de fond: l'étendue des pouvoirs conférés aux magistrats par leurs lettres de commission, le peu de considération qu'ils portèrent au cahier préparatoire dressé par les praticiens locaux, la promptitude des commissaires à faire taire les opposants en les renvoyant à se pouvoir devant le parlement à la saint Martin suivante ${ }^{28}$. Aux yeux de l'avocat Pierre Versoris, qui plaidait pour Françoise d'Ailly, la sœur, le point essentiel, que René Filhol n'a pas mis en lumière, était la question du serment exigé des participants. Ce serment comportait deux choses:

[...] la première que, toute affection cessant, ilz rapporteront au vray ce qu'ilz ont veu le temps passé observer pour coustume, et la seconde que, pour le bien public, ilz diront en quoy ilz trouvent lad. coustume dure et desraisonnable pour la corriger.

Serment qui fait bien fort a notte (soubz correction) [concluait Versoris : à ses yeux], ceulx qui ont juré n'ont pas fait ne eus moyen et loisir de faire ne l'un ne l'autre des deux choses qu'ilz avoyent promises et juréez ${ }^{29}$.

17 C'est Claude Mangot, avocat de la veuve et des enfants de Charles d'Ailly, neveux du vidame, qui se trouvait chargé de justifier l'œuvre des commissaires. Il réfuta, outre des questions de fait, l'argument de Versoris qui assimilait les opérations de rédaction et de réformation, car tousjours, dit Mangot, le premier ceuvre est le plus difficile. Il justifiait ainsi ce qui pouvait apparaître comme la hâte excessive des commissaires qui présumaient, sinon de leur virtuosité, au moins de leur capacité pédagogique. Mais tous les avocats des plaignants (Jean de Sainct Meloir, pour le duc d'Aumale, Louis Buisson, pour le cardinal de Guise...) mettaient l'accent sur l'essentiel : tout s'était passé comme si les commissaires avaient donné loi de leur propre chef aux Picards, et non pas simplement réformer la 
coutume de 1507, comme le portait leur commission, ce en quoi ils s'étaient arrogé plus de puissance que le Roy et tout son Conseil privé ${ }^{30}$. La question du consentement des états était formellement essentielle, d'où l'insistance sur la question $\mathrm{du}$ serment ${ }^{31}$, car la réformation engageait les Picards pour les générations à venir. Louis Buisson plaida qu'il vaudrait mieux les assembler cinquante fois que de leur donner une loy contre leur gré et consentement $^{32}$. Ces accusations ne sont pas à prendre pour argent comptant, mais il faut noter l'embarras de l'avocat général Baptiste Dumesnil qui ne prit pas la défense de ses collègues avec l'énergie qu'on aurait pu attendre.

Tout porte à croire que les commissaires, confrontés à de telles récriminations, s'estimaient investis de la science du droit vis-à-vis des demi-savants qu'étaient, à leurs yeux, les praticiens du siège et de ces simples sujets privilégiés qu'étaient les députés, toujours soupçonnés de supporter des intérêts particuliers qu'il fallait retrancher au nom $\mathrm{du}$ bien commun et de l'autorité royale. Les juristes savants ne s'opposaient pas seulement aux rustiques, mais aussi aux représentants. La solidarité des commissaires réformateurs tournait à la complicité. L'affaire qui occupe tant de place dans les registres de l'année 1568 fut simplement enterrée : les parties furent appointées, Françoise d'Ailly mourut, les poursuites ne furent pas entretenues et un arrêt définitif du 7 septembre 1571 mit l'appel à néant et autorisa l'impression des coutumes réformées d'Amiens ${ }^{33}$.

\section{Les solidarités internes entre les commissaires rédacteurs et réformateurs}

19 L'ordonnance de Montilz-lez-Tours et encore les lettres patentes de 1493 prévoyaient que la coutume fût rédigée par l'assemblée des états sous la direction des officiers des bailliages. Lors d'une première reprise en main, le roi avait désigné une commission permanente de huit membres pour procéder aux rédactions $(1497)^{34}$. Mais Louis XII préféra nommer des commissaires particuliers pour chaque circonscription. Cette solution n'empêcha pourtant pas des spécialistes d'émerger de la masse des magistrats, surtout lors des réformations. Le temps des rédactions et le temps des réformations semblent marquer par une opposition nette quant au choix des commissaires.

1. En gros, à la fin $d u x^{e}$ siècle et au début $d u x v l^{e}$ siècle, étaient envoyés dans les provinces des conseillers qui en étaient originaires et qui connaissaient d'autant mieux les coutumes locales qu'ils étaient les rapporteurs attitrés des procès venant en appel devant les parlements depuis les sièges locaux. La présence de ces enfants du pays qui avaient réussi était rassurante pour les notables de l'assemblée : ils étaient sortis de leurs rangs, avaient parfois compté parmi les juges ou les praticiens du siège, ils étaient leurs parents et alliés ou les clients des grands seigneurs qui patronnaient la province. Leurs relations dans le pays assuraient la bonne fin des négociations éventuelles sur les oppositions et procuraient des soutiens de clientèle, si besoin était. Ainsi Jean (Le) Prévost, qui rédigea à Blois en 1523, n'appartenait-il pas seulement à une famille blésoise, il comptait parmi les serviteurs les plus anciens des ducs d'Orléans et du feu roi Louis XII. L'obscur conseiller clerc Pierre Mathé, qui officie à Bourges en 1539, était doyen du chapitre de la cathédrale et fils d'un avocat et conseiller à la prévôté ou au bailliage ${ }^{35}$. En 1534, Guillaume Bourgoing est envoyé à Nevers, parce qu'il était nivernais et figurait parmi les vieux clients des Clèves. Il n'en était pas ainsi des seuls conseillers. Le procureur général Nicolas Thibault est commissaire en 1539 à Senlis dont il était originaire. En 
Auvergne, le futur chancelier Duprat aurait dû être accompagné d'un autre auvergnat, Louis Doreille, chanoine de Clermont, selon l'ordonnance royale du 19 décembre 1508. Mais finalement, à Lyon, le 31 mai 1510, c'est le conseiller champenois Picot qui fut chargé d'assister le président Duprat, parce qu'il se trouvait déjà sur les lieux ${ }^{36}$. Prétexte un peu mince, mais il suffisait sans doute qu'un des commissaires eût bénéficié d'une bonne implantation locale.

2. Lors des réformations, la solution provinciale semble largement abandonnée. Lors des rédactions déjà, on observe l'intense activité d'un tandem constitué par les présidents Barme et Baillet, deux magistrats spécialisés, tous deux d'ancienne famille parisienne. C'était vraisemblablement la compétence et l'autorité qu'on leur prêtait en ces matières qui amenèrent le conseil du roi à avoir aussi souvent recours à leurs services ${ }^{37}$. Ces mêmes critères ont conduit à confier à un trio omniprésent et voyageur l'œuvre de réformation : Christophe de Thou, Barthélemy Faye et Jacques Viole. Ces juges furent des activistes de la réforme: la reprise de l'activité de codification a été mise en relation par Etienne Pasquier avec l'édit du semestre (1554) qui réduisait les magistrats à l'inactivité six mois de l'année ${ }^{38}$. René Filhol a remarqué qu'ils étaient inséparables et que leur activité de commissaires ne concernait pas seulement les coutumes, puisque le souverain leur confia le procès du vidame de Chartres ou celui du prince de Condé3 ${ }^{39}$. Le président de Thou était le plus âgé et son leadership était reconnu, mais Viole (1543) et Faye (1542) étaient plus anciennement magistrats que lui qui avait si longtemps brillé au barreau quoiqu'il ait été fils de président. Les trois commissaires disparurent dans les mêmes années. De Thou en 1582, Jacques Viole en 1584 et Barthélemy Faye dès $1581^{40}$. La solidarité qui les animait reposait aussi sur des relations privées : on peut dire qu'ils travaillaient en famille.

3. Les trois hommes (voir les tableaux généalogiques en annexe) n'étaient en effet pas seulement unis par la science du droit et par leur proximité d'esprit, mais aussi par la parenté et l'alliance. Les de Thou et les Viole étaient originaires d'Orléans où ils appartenaient au patriciat urbain. Mais, alors que les de Thou étaient marchands pelletiers au $\mathrm{Xv}^{\mathrm{e}}$ siècle, les Viole exerçaient des professions juridiques dès le début du siècle. Aignan Viole devint avocat du roi à la cour des Aides de Paris (il mourut en 1473). Il avait pour nièce Marie Viole, épouse du marchand Jacques I de Thou. L'office d'avocat du roi aux Aides passa à Jacques II, fils de Jacques I de Thou, avant de revenir aux petits-fils d'Aignan Viole, l'aîné, Jean, père de Jacques, le commissaire des réformations, puis le troisième Pierre, qui fut prévôt des marchands de Paris et connut très bien à l'Hôtel de Ville Augustin de Thou, père de Christophe. Le souvenir de l'origine commune était donc largement entretenu par des relations politiques centrées sur l'exercice (et peut-être la résignation) d'offices dans les mêmes compagnies et sur la gestion du corps de ville parisien, auquel, à cette génération, les de Thou et les Viole témoignaient un très vif attachement.

Le Lyonnais Barthélemy Faye était cousin au sixième degré de la mère de Jacques Viole, fille de Blanche Thurin et de Jean Caille, juge des ressorts de Lyon ${ }^{41}$. Cette parenté fut rajeunie par une alliance très proche, puisque $B$. Faye épousa en 1542 la sœur de Jacques Viole. Il n'empêche que, malgré les origines ou alliances lyonnaises de Faye et de Viole, ce trio de commissaires paraît caractérisé par un esprit bien parisien, celui de la capitale du royaume qui se pensait comme sa tête. Ce sentiment pouvait répondre à certaines orientations de la politique royale. La phase de rédaction semble plutôt décentralisatrice; la phase des réformations semble marquée par une volonté plus centralisatrice. C'est bien ce que traduit l'évolution de la sociologie des commissaires, telle qu'elle a été décrite au 
début de ce développement. On pourrait dresser une prosopographie détaillée des commissaires, mais il n'est pas sûr que cela apporterait une meilleure compréhension de leur travail.

24 Les conclusions que je pourrais présenter sont celles de Filhol et d'Olivier Martin. Les contemporains, surtout les avocats et praticiens des sièges, couvraient d'éloges les commissaires des parlements. Il apparait que leur travail répondit à un besoin social largement ressenti. Il est difficile de toujours cerner leur rôle personnel ou de généraliser. Mais ils comptèrent, à n'en pas douter, parmi les hommes qui influencèrent le plus le devenir du droit dans l'ancienne France.

\section{Tableaux généalogiques}

\section{'Trableateaul 1.}

\section{De Thou et Violle}

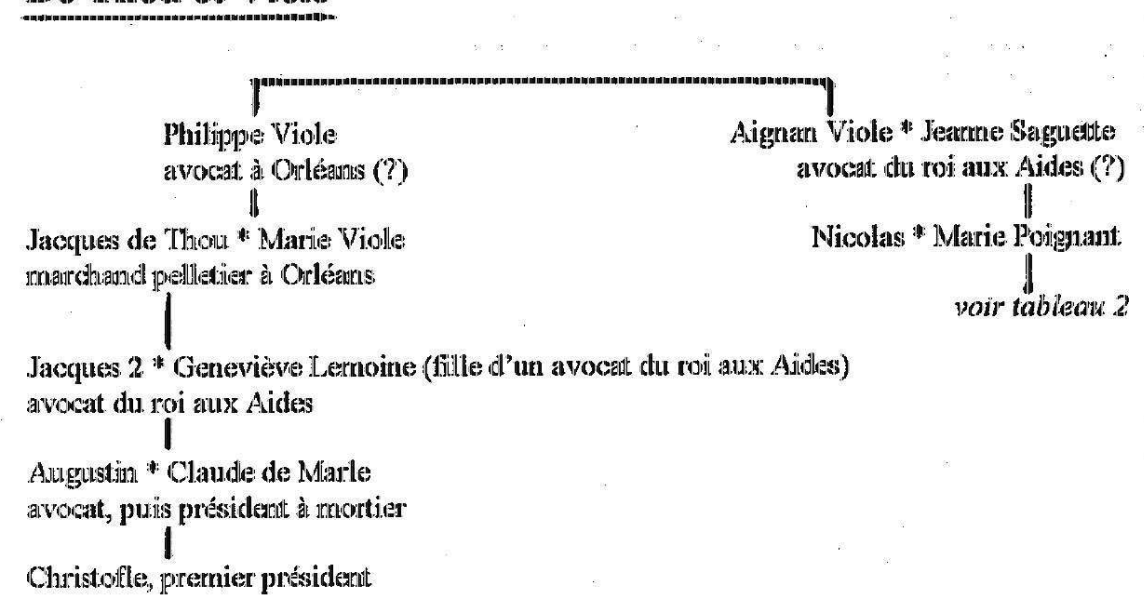

(voir Viole at Farye page surivante)

(voir Viole et Faye page suivante) 


\title{
T'Tableatu 2
}

\author{
Viole et Faye

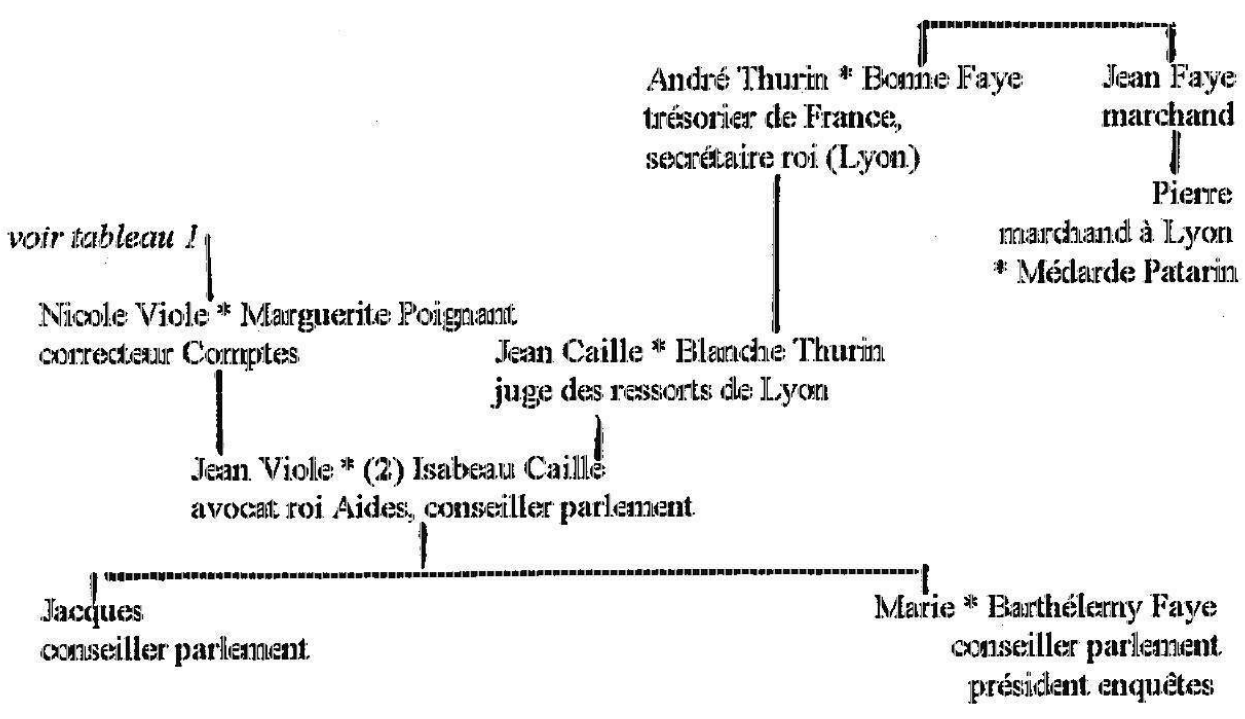

\section{NOTES}

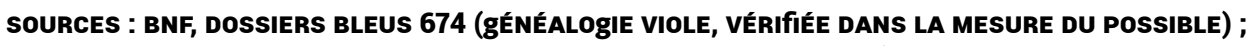
AN, MIN. CENTR. DES NOTAIRES PARISIENS, CVII 93, 24 FÉVRIER 1573, (INV. APRÈS dÉcÈS de NICOLAS VIOLE (UN COUSIN), ET XVIII 214, 15 FÉVRIER 1610, INV. APRÈS DÉCÈs DE JACQUES II VIOLE, fiLS DU COMPAgNON DE CHRISTOFLe de thou. OLIVIER PONCET, OP. CIT., TABL. génÉAL. 8 et 9 (FAYE), P. 423-424.

1. Il est difficile d'apporter du nouveau aux réflexions sur la rédaction et les commissaires de René Filhol Le Premier Président Christofle de Thou et la réformation des coutumes, Paris, 1937, et de François Olivier-Martin, Histoire de la coutume de la prévôté et vicomté de Paris, Paris, 3 vol., 1922-1930, réimpression complétée par des additions bibliographiques, Paris, 2 vol., 1972.

2. Françoise Autrand, Naissance d'un grand corps de l'État. Les gens du Parlement de Paris 1345-1454, Paris, PUF, 1981. Mais la magistrature du $\mathrm{XVI}^{\mathrm{e}}$ siècle a beaucoup changé par rapport aux parlementaires de la fin du Moyen Âge .

3. William J. Bouwsma, "Lawyers and Early Modern Culture ", American Historical Review, 78/2, 1973, p. 303-327. Robert Descimon, "Juristes, science du droit et pouvoir d'État au temps de Galilée », Celebrazioni del IV Centenario, IV, Tribute to Galileo in Padoua, Trieste, 1995, p. 103-127.

4. On rappellera les évidentes conclusions de Jack Goody, Entre l'oralité et l'écriture, Paris, 1994 (Cambridge, 1993) : «les opérations formelles [...] peuvent aussi aider à briser la croûte de la pensée routinière en mettant au jour les «contradictions ", les «illogismes ", les "absences de suite », les ambiguités dans les catégories et ainsi de suite, sur lesquelles on glisse plus facilement dans la parole », p. 295. 
5. Guy Coquille, Commentaires de la coutume de Nivernois, dans Euvres, éd. 1723, Bordeaux, t. II, p. 313 : «Les coustumes prennent force de loy par l'autorisation que le Roy en fait par ses commissaires".

6. Antonio Manuel Hespanha, «Savants et rustiques. La violence douce de la raison juridique », Jus commune, 10, 1983, p. 1-47.

7. René Filhol, op. cit., p. 215-218 : «sur chacun des textes réformés par Christofle de Thou allait s'abattre une véritable nuée de commentateurs qui, en d'autres circonstances, eussent fait des Glossateurs et des Bartolistes impénitents ».

8. René Filhol, op. cit., p. 171-174. Jean-Louis Thireau, Charles Dumoulin (1500-1566), Genève, 1980. Le rôle de Charles Dumoulin fut essentiel en ce que sa doctrine influença directement les conceptions des magistrats réformateurs. C'est d'ailleurs toujours le point de vue de Charles Du Moulin qu'adoptent les historiens du droit «privé » quand ils tiennent à en donner un exposé unitaire : "c'est le droit familial dans son ensemble qui s'est imposé à la féodalité », concluait François Olivier-Martin, op. cit., t. II, p. 142.

9. J. Russell Major, From Renaissance Monarchy to Absolute Monarchy: French Kings, Nobles and Estates, Baltimore, 1994, donne la version la plus récente d'une vision de l'évolution politique de la France que cet auteur a développée depuis les années 1960, sans toujours entraîner l'adhésion, mais en forçant les historiens français à réfléchir sur les schémas canoniques qu'ils ont des difficultés à remettre en question.

10. William Church, Constitutional Thought in Sixteenth-Century France, Cambridge, 1941 ; Robert Knecht, Un prince de la Renaissance. François $I^{\text {er }}$ et son royaume, Paris, 1998 (1994), p. 521-545, qui, tout en parlant d'« absolutisme limité », combat les thèses de J. Russell Major.

11. Albert Babeau, «La représentation du tiers état aux assemblées pour la rédaction des coutumes au XVI ${ }^{e}$ siècle ", Revue historique, XXI, 1883, p. 91-100, parlait d'une « sorte de révolution latente en faveur du peuple des campagnes ». La vague anti-féodale qu'animèrent les juristes au $\mathrm{XVI}^{\mathrm{e}}$ siècle présageait par bien des côtés ce que fut l'action des avocats dans les premières années de la Révolution française.

12. René Filhol, op. cit., p. 155-158. L'avocat Louis de Saint Yon, représentant du tiers état lors de la réformation de la coutume de Paris (1580), a dressé une double liste des droits seigneuriaux «tollus » lors de la rédaction de 1510, puis lors de la réformation.

13. René Filhol, op. cit., p. 93.

14. Robert Jacob, Les Époux, le seigneur et la cité : coutumes et pratiques matrimoniales des bourgeois et paysans de France du Nord au Moyen Âge, Bruxelles, 1990, p. 11-26.

15. René Filhol, op.cit., p. 125-128, qui commente les assertions et analyses de Guy Coquille, Claude Dupré, René Choppin, en particulier à propos de la coutume de Berry réformée par Lizet en 1539.

16. On se reportera aux réflexions générales de Dominique Julia et Jacques Revel, Histoire sociale des populations étudiantes, t. II, Paris, 1989, p. 33-105.

17. Une telle hypothèse ne rejoint nullement l'allégation aventurée d'une « relégation du droit romain " familière à Blandine Barret-Kriegel, par exemple, Les Chemins de l'État, Paris, 1986, p. 15-31 («L'esprit du droit romain et l'Etat moderne ») et p. 93-120 (« La politique juridique de la monarchie »).

18. Vincenzo Piano Mortari, Diritto romano e diritto nazionale in Francia nel secolo XVI, Milan, 1962, 180 p. ; Myron P. Gilmore, Argument from Roman Law in Political Thought, Harvard, 1941.

19. René Filhol, op.cit., p.63-71: «la coutume prenait l'allure d'une convention, d'une transaction entre les trois états », p. 66.

20. Ce développement est fondé sur Donald Kelley, "Jurisconsultus Perfectus: The Lawyer as Renaissance Man ", Journal of the Warburg and Courtauld Institute, 51, 1988, p. 84-102, repris dans The Writing of History and the Study of Law, Londres, Variorum, 1997, $\mathrm{n}^{\circ}$ 13. "Second Nature »: The Idea of Custom in European Law, Society and Culture », The Transmission of Culture in Early Modern 
Europe, Anthony Grafton et Ann Blair (éd.), Philadelphie, 1990, p. 131-172. "Vera Philosophia ». The Philosophical Significance of Renaissance Jurisprudence ", Journal of the History of Philosophy, 14,1976, p. 267-279.

21. Guy Fourquin, «Le droit parisien de la fin du Moyen Âge : droit des "notables » ", Études d'histoire du droit parisien, Paris, 1970, p. 375-395 (synthèse encore précieuse).

22. Françoise Autrand, «" Tous parens, amis et affins »: Le groupe familial dans le milieu de robe parisien au XV siècle », Commerce, finance et société (XI ${ }^{e}-\mathrm{XVI}^{e}$ siècles), Paris, 1993, p. 347-357, montre $\mathrm{qu}^{\prime} \mathrm{au} \mathrm{xv}^{\mathrm{e}}$ siècle encore, les gens du parlement ne faisaient pas passer par le patrilignage de type nobiliaire leur projet de perpétuation familiale.

23. Mes propres observations dans les actes notariés recoupent les analyses de René Filhol, op. cit., p. 224-227.

24. Le jugement de Colbert et de Pussort était aussi négatif sur les grandes ordonnances du XVI siècle que sur les rédactions.

25. René Filhol, op. cit., p. 84-86.

26. René Filhol, op. cit., p. 94-121.

27. Arch. nat., X 1A 5018, 20 août 1568.

28. Ibid., fo 331 : « on les print a partye en leurs noms, comme on feist ung advocat, le plus ancien du siege $" . .$.

29. Ibid., fo $330 \mathrm{v}^{\mathrm{o}}-331$. René Filhol, op. cit., p. 98, donne la suite de l'argument.

30. René Filhol, op. cit., p. 104.

31. Paolo Prodi, Il sacramento del potere. Il giuramento politico nella storia costituzionale dell'Occidente, Bologne, 1992, IV, La società « giurata » del tardo Medioevo, p. 161-225.

32. René Filhol, op. cit., p. 119.

33. René Filhol, op. cit., p. 118.

34. François Olivier-Martin, «Un document inédit sur les travaux préparatoires de l'ancienne coutume de Paris ", Nouvelle Revue historique de droit français et étranger, XLII, 1918, p. 192-227.

35. Edouard Maugis, Histoire du parlement de Paris, Paris, 1916 (reprint Genève, 1977), t. III, p. 169. En général, les renseignements biographiques sont tirés de cet ouvrage, confirmés par mes recherches personnelles.

36. Albert Buisson, Le Chancelier Antoine Duprat, Paris, 1935, p. 79-81.

37. Jean Yver, «Le président Thibault Baillet et la rédaction des coutumes (1496-1514) », Revue historique de droit français et étranger, t. LXIV, 1986, p. 19-42.

38. Estienne Pasquier, Lettres, VII, 10, dans Euvres, Amsterdam, 1723, t. II, col. 185, qui contient un éloge funèbre circonstancié du premier président.

39. René Filhol, op. cit., p. 37.

40. Sa mort fut vraiment lamentable : il s'endormit dans son étude, alors qu'il lisait, et tomba dans son feu : Olivier Poncet, Pomponne de Bellièvre (1529-1607), Paris, 1998, p. 149.

41. Olivier Poncet, tableaux généalogiques 8 et 9 . 